\title{
Existence and multiplicity of solutions for Kirchhof-type problems with Sobolev-Hardy critical exponent
}

Hongsen Fan ${ }^{1 *}$ and Zhiying Deng ${ }^{1}$

"Correspondence:

991819530@qq.com

'School of Science, Chongqing

University of Posts and

Telecommunications, Chongqing

400065, P.R. China

\begin{abstract}
In this paper, we discuss a class of Kirchhof-type elliptic boundary value problem with Sobolev-Hardy critical exponent and apply the variational method to obtain one positive solution and two nontrivial solutions to the problem under certain conditions.
\end{abstract}

MSC: 35B38; 35G99

Keywords: Kirchhof-type equation; Positive solution; Sobolev-Hardy critical exponent; Mountain pass theorem

\section{Introduction and main results}

In this paper, we investigate the following Kirchhof-type problem with Soblev-Hardy critical exponent:

$$
\begin{cases}\left\{a+b\left[\int_{\Omega}\left(|\nabla u|^{2}-\mu \frac{u^{2}}{|x|^{2}}\right) \mathrm{d} x\right]^{\frac{2-s}{2}}\right\}\left(-\Delta u-\mu \frac{u}{|x|^{2}}\right) & \\ =\frac{|u|^{2^{*}(s)-2} u}{|x|^{s}}+f(x, u) & \text { in } \Omega, \\ u=0, & \text { on } \Omega \Omega,\end{cases}
$$

where $\Omega$ is a smooth bounded domain in $\mathbb{R}^{3}, 0 \in \Omega, a, b>0,0 \leq s<2, \mu \in[0,1 / 4), 2^{*}(s)=$ $6-2 s$ is the Sobolev-Hardy critical exponent, and $f(x, t): \bar{\Omega} \times \mathbb{R}$ is a continuous real function.

Equation (1.1) is called a Schrödinger equation of Kirchhoff type due to the presence of the term $b\left[\int_{\Omega}\left(|\nabla u|^{2}-\mu u^{2}|x|^{-2}\right) \mathrm{d} x\right]^{(2-s) / 2}$. When $\mu=0$ and $s=1$, it appears in the following classical Kirchhoff type equation:

$$
\begin{cases}-\left(a+b \int_{\Omega}|\nabla u|^{2} \mathrm{~d} x\right) \Delta u=k(x, u) & \text { in } \Omega, \\ u=0 & \text { on } \partial \Omega,\end{cases}
$$

(c) The Author(s) 2021. This article is licensed under a Creative Commons Attribution 4.0 International License, which permits use, sharing, adaptation, distribution and reproduction in any medium or format, as long as you give appropriate credit to the original author(s) and the source, provide a link to the Creative Commons licence, and indicate if changes were made. The images or other third party material in this article are included in the article's Creative Commons licence, unless indicated otherwise in a credit line to the material. If material is not included in the article's Creative Commons licence and your intended use is not permitted by statutory regulation or exceeds the permitted use, you will need to obtain permission directly from the copyright holder. To view a copy of this licence, visit http://creativecommons.org/licenses/by/4.0/. 
related to the stationary analogue of the equation

$$
u_{t t}-\left(a+b \int_{\Omega}|\nabla u|^{2} \mathrm{~d} x\right) \Delta u=f(x, t)
$$

which was first proposed by Kirchhoff [1] as an extension of the classical D'Alembert wave equation for free vibrations of elastic strings. Equation (1.2) has aroused widespread concern after the work of Lions [2], which proposes a function analysis framework. After that, many interesting results have been obtained such as [3-9]. For instance, $\mathrm{Xu}$ and Chen [10] studied Kirchhoff-type equations with a general nonlinearity in the critical growth. Under certain conditions, the existence of ground state solutions were proved by using variational methods. In particular, they do not use the classical Ambrosetti-Rabinowitz condition. Fiscella et al. [11] dealt with the existence of nontrivial solutions for critical Hardy-Schrd̈inger-Kirchhoff systems driven by the fractional $p$-Laplacian operator. The existence was derived as an application of the mountain pass theorem and the Ekeland variational principle. The authors extend the existence results recently obtained for fractional systems to entire solutions with critical nonlinear terms and generalized the systems driven by the $p$-Laplacian operator to the fractional Hardy-Schrödinger-Kirchhoff case. Xiang and Vicentiu [12] investigated the existence of solutions for critical SchrödingerKirchhof-type systems driven by nonlocal integro-differential operators. By applying the mountain pass theorem and Ekeland's variational principle, the existence and asymptotic behavior of solutions for the problem under some suitable assumptions were obtained. A distinguished feature of their paper is that the systems are degenerate, that is, the Kirchhoff function could vanish at zero. This is the first time of exploiting the existence of solutions for fractional Schrödinger-Kirchhoff systems involving critical nonlinearities in $R^{N}$.

In the case $k(x, u)=f(x, u)+u^{5}$, Xie et al. [6] studied the nondegenerate and degenerate cases and proved the existence and multiplicity of solutions by using the Brezis-Lieb lemma and mountain pass theorem. Naimen [8] further discussed this problem in the case of $k(x, u)=\mu g(x, u)+u^{5}$ under different conditions of $g(x, u)$ and $\mu \in \mathbb{R}$. In the meantime, the results were expanded in [6] by establishing the existence and nonexistence of positive solutions by using the second concentration compactness lemma and mountain pass theorem.

Problem (1.1) in the case of $a=1$ and $b=0$ can be reduced to the classic semilinear elliptic problem with critical exponents, for which the existence and multiplicity of solutions was proved by Ding and Tang [9].

Inspired by the results of the above paper, the purpose of this paper is to consider the existence and multiplicity of solutions to problem (1.1). The main difficulty in this paper is that it contains the Sobolev-Hardy critical exponent term, which leads to the energy functional not satisfying the Palais-Smale condition.

In order to state our main results, let $F(x, u)=\int_{0}^{u} f(x, t) \mathrm{d} t$. We introduce the following assumptions:

$\left(f_{1}\right) f \in C\left(\Omega \times \mathbb{R}^{+}, \mathbb{R}\right)$, and $\lim _{t \rightarrow 0^{+}} \frac{f(x, t)}{t}=0, \lim _{t \rightarrow+\infty} \frac{f(x, t)}{t^{5-2 s}}=0$ uniformly for a.e. $x \in \Omega$.

$\left(f_{2}\right)$ There exists a constant $\rho>\max \left\{\frac{6}{1+\sqrt{1-4 \mu}}, 6-\sqrt{1-4 \mu}\right\}$ such that $0<\rho F(x, t) \leq f(x, t) t$ for all $x \in \bar{\Omega}, t \in \mathbb{R}^{+} \backslash\{0\}$.

$\left(f_{3}\right) f \in C(\Omega \times \mathbb{R}, \mathbb{R})$, and $\lim _{t \rightarrow 0} \frac{f(x, t)}{t}=0, \lim _{t \rightarrow+\infty} \frac{f(x, t)}{t^{5-2 s}}=0$ uniformly for a.e. $x \in \Omega$.

$\left(f_{4}\right)$ There exists a constant $\rho>\max \left\{\frac{6}{1+\sqrt{1-4 \mu}}, 6-\sqrt{1-4 \mu}\right\}$ such that $0<\rho F(x, t) \leq f(x, t) t$ for all $x \in \bar{\Omega}, t \in \mathbb{R} \backslash\{0\}$. 
Now our main results are as follows.

Theorem 1.1 Let $f(x, t)$ satisfy $\left(f_{1}\right)$ and $\left(f_{2}\right)$. Then problem (1.1) has at least one positive solution.

Theorem 1.2 Let $f(x, t)$ satisfy $\left(f_{3}\right)$ and $\left(f_{4}\right)$. Then problem (1.1) has at least two distinct nontrivial solutions.

Remark 1.1 We added the Hardy and Sobolev-Hardy terms in equation (1.1) on the basis of [6]. We overcome the compactness problem with concentration compactness principle. Lei [7] studied another special case of problem (1.1) with $f(x, u)=\lambda f(x)|u|^{q-2} u|x|^{-\beta}$ for a suitable function $f(x)$ and $1<q<2$. By using the Nehari manifold and fibering maps, they obtained two positive solutions. We observe that the term $\lambda f(x)|u|^{q-2} u|x|^{-\beta}$ has to be a homogeneous function; however, it does not satisfy the assumptions we give in this paper.

The rest of this paper is organized as follows. In Sect. 2, we give some preliminary results. In Sect. 3, we establish the proofs of our main results.

\section{Preliminaries}

In this part, we give some information to support this paper. Otherwise stated, $C, C_{0}, C_{1}, \ldots$ represent positive constants, and " $\rightarrow$ " and " $\rightarrow$ " represent the strong convergence and weak convergence in the corresponding space, respectively. Let $H_{0}^{1}(\Omega)$ be the usual Hilbert space endowed with the usual inner product and norm

$$
(u, v)_{H_{0}^{1}(\Omega)}=\int_{\Omega} \nabla u \nabla v \mathrm{~d} x \text { and }\|u\|_{H_{0}^{1}(\Omega)}=\left(\int_{\Omega}|\nabla u|^{2} \mathrm{~d} x\right)^{\frac{1}{2}}
$$

By the well-known Hardy inequality [13]

$$
\int_{\Omega} \frac{u^{2}}{|x|^{2}} \mathrm{~d} x \leq 4 \int_{\Omega}|\nabla u|^{2} \mathrm{~d} x
$$

we deduce that

$$
(u, v)=\int_{\Omega}\left(\nabla u \nabla v-\mu \frac{u v}{|x|^{2}}\right) \mathrm{d} x \quad \text { and } \quad\|u\|=\left[\int_{\Omega}\left(|\nabla u|^{2}-\mu \frac{u^{2}}{|x|^{2}}\right) \mathrm{d} x\right]^{\frac{1}{2}},
$$

respectively, which are equivalent to the usual inner product and norm on $H_{0}^{1}(\Omega)$ for any $\mu \in[0,1 / 4)$.

We also define the best Sobolev-Hardy constant

$$
S \triangleq \inf _{u \in H_{0}^{1}(\Omega) \backslash\{0\}} \frac{\int_{\Omega}\left(|\nabla u|^{2}-\mu \frac{u^{2}}{|x|^{2}}\right) \mathrm{d} x}{\left(\int_{\Omega} \frac{|u|^{*}(s)}{|x|^{S}} \mathrm{~d} x\right)^{\frac{2}{2^{*}(s)}}} .
$$

From Lemma 2.2 in [14] we find that $S$ is independent of $\Omega$, and when $\Omega=\mathbb{R}^{3}$, it is obtained by the functions

$$
y_{\epsilon}(x)=\left[\frac{2 \epsilon(3-s)(\bar{\mu}-\mu)}{\sqrt{\bar{\mu}}}\right]^{\frac{\sqrt{\mu}}{2-s}} /\left[|x|^{\sqrt{\bar{\mu}}-\sqrt{\bar{\mu}-\mu}}\left(\epsilon+|x|^{\frac{(2-s) \sqrt{\bar{\mu}-\mu}}{\sqrt{\bar{\mu}}}}\right)^{\frac{3-s}{2-s}}\right]
$$


for all $\epsilon>0$ and $\bar{\mu}=1 / 4$. In addition, the function $y_{\epsilon}(x)$ is the solution to the equation

$$
-\Delta u-\mu \frac{u}{|x|^{2}}=\frac{|u|^{2^{*}(s)-2} u}{|x|^{s}} \quad \text { in } \mathbb{R}^{3} \backslash\{0\}
$$

Since $0 \in \Omega$, let $R_{0}$ be a positive constant such that $B_{2 R_{0}}(0) \subset \Omega$. We take a cut-off function $\eta(x) \in C_{0}^{\infty}(\Omega)$ such that $\eta(x)=1$ for $|x| \leq R_{0}, \eta(x)=0$ for $|x|>2 R_{0}$, and $0 \leq \eta(x) \leq 1$ otherwise. Let $C_{\epsilon}=\left[\frac{2 \epsilon(3-s) \sqrt{\bar{\mu}-\mu}}{\sqrt{\bar{\mu}}}\right]^{\frac{\sqrt{\mu}}{2-s}}, \gamma_{1}=\sqrt{\bar{\mu}}-\sqrt{\bar{\mu}-\mu}, \gamma_{2}=\sqrt{\bar{\mu}}+\sqrt{\bar{\mu}-\mu}$, and $U_{\epsilon}(x)=\frac{y_{\epsilon}(x)}{C_{\epsilon}}$. Suppose

$$
\begin{aligned}
& U_{\epsilon}(x)=\frac{1}{\left[|x|^{\left.\frac{2-s}{2} \frac{\gamma_{1}}{\sqrt{\bar{\mu}}}+|x|^{\frac{2-s}{2}} \frac{\gamma_{2}}{\sqrt{\bar{\mu}}}\right]^{\frac{2 \sqrt{\mu}}{2-s}}},\right.} \\
& u_{\epsilon}(x)=\eta(x) U_{\epsilon}(x)=\frac{\eta(x)}{\left[\epsilon|x|^{\frac{2-s}{2}} \frac{\gamma_{1}}{\sqrt{\bar{\mu}}}+|x|^{\frac{2-s}{2} \frac{\gamma_{2}}{\sqrt{\bar{\mu}}}}\right]^{\frac{2 \sqrt{\mu}}{2-s}}}, \\
& v_{\epsilon}(x)=\frac{u_{\epsilon}(x)}{\left(\int_{\Omega} \frac{\left|u_{\epsilon}(x)\right|^{* *(s)}}{\left.|x|\right|^{s}} \mathrm{~d} x\right)^{\frac{1}{2^{*}(s)}},}
\end{aligned}
$$

so that $\left\|v_{\epsilon}(x)\right\|_{L^{2^{*}(s)}\left(\Omega,|x|^{-s}\right)}^{2^{*}(s)}=\int_{\Omega}\left|v_{\epsilon}(x)\right|^{2^{*}(s)}|x|^{-s} \mathrm{~d} x=1$. Then we have the following results [14]:

$$
\begin{aligned}
& \left\|v_{\epsilon}(x)\right\|^{2}=S+O\left(\epsilon^{\frac{1}{2-s}}\right), \\
& \int_{\Omega}\left|\nu_{\epsilon}\right|^{q} \mathrm{~d} x= \begin{cases}O\left(\epsilon^{\frac{q \sqrt{\mu}}{2-s}}\right), & 1 \leq q<\frac{3}{\sqrt{\bar{\mu}}+\sqrt{\bar{\mu}-\mu}}, \\
O\left(\epsilon^{\frac{q \sqrt{\mu}}{2-s}}|\ln \epsilon|\right), & q=\frac{3}{\sqrt{\bar{\mu}}+\sqrt{\bar{\mu}-\mu}}, \\
O\left(\epsilon^{\frac{\sqrt{\bar{\mu}}(3-q \sqrt{\bar{\mu}})}{(2-) \sqrt{\bar{\mu}-\mu}}}\right), & \frac{3}{\sqrt{\bar{\mu}}+\sqrt{\bar{\mu}-\mu}}<q<6 .\end{cases}
\end{aligned}
$$

Now we define the functional $I$ on $H_{0}^{1}(\Omega)$ by

$$
I(u)=\frac{a}{2}\|u\|^{2}+\frac{b}{4-s}\|u\|^{4-s}-\frac{1}{2^{*}(s)} \int_{\Omega} \frac{u^{2^{*}(s)}}{|x|^{s}} \mathrm{~d} x-\int_{\Omega} F(x, u) \mathrm{d} x .
$$

Obviously, the functional $I$ belongs to the class $C^{1}\left(H_{0}^{1}(\Omega), \mathbb{R}\right)$. Furthermore,

$$
\begin{aligned}
\left\langle I^{\prime}(u), v\right\rangle= & a \int_{\Omega}\left(\nabla u \nabla v-\mu \frac{u v}{|x|^{2}}\right) \mathrm{d} x+b\|u\|^{2-s} \int_{\Omega}\left(\nabla u \nabla v-\mu \frac{u v}{|x|^{2}}\right) \mathrm{d} x \\
& -\int_{\Omega} \frac{u^{2^{*}(s)-1} v}{|x|^{s}} \mathrm{~d} x-\int_{\Omega} f(x, u) v \mathrm{~d} x, \quad \forall v \in H_{0}^{1}(\Omega) .
\end{aligned}
$$

\section{Proofs of our main results}

In this section, we consider the existence and multiplicity of solutions to problem (1.1). We first verify that the functional $I(u)$ satisfies the local $(P S)$ condition. 
Lemma 3.1 Let $f(x, t)$ satisfy $\left(f_{1}\right)$ and $\left(f_{2}\right)$. Suppose $c \in\left(0, \Lambda_{0}\right)$, where

$$
\begin{aligned}
\Lambda_{0}= & \frac{a(2-s)}{2(3-s)} S\left[\frac{b S^{\frac{4-s}{2}}+\sqrt{b^{2} S^{4-s}+4 a S}}{2}\right]^{\frac{2}{2-s}} \\
& +\frac{b(2-s)}{2(3-s)(4-s)} S\left[\frac{b S^{\frac{4-s}{2}}+\sqrt{b^{2} S^{4-s}+4 a S}}{2}\right]^{\frac{4-s}{2-s}} .
\end{aligned}
$$

Then $I(u)$ satisfies the local $(P S)_{c}$ condition.

Proof Suppose that $\left\{u_{n}\right\}$ is a $(P S)_{c}$ sequence. Then, for $c \in\left(0, \Lambda_{0}\right)$,

$$
I\left(u_{n}\right) \rightarrow c, \quad I^{\prime}\left(u_{n}\right) \rightarrow 0 \quad \text { as } n \rightarrow \infty
$$

First, we prove that $\left\{u_{n}\right\}$ is a bounded sequence. From (3.1) we have

$$
\begin{aligned}
1+c+o(1)\left\|u_{n}\right\| \geq & I\left(u_{n}\right)-\frac{1}{\theta}\left\langle I^{\prime}\left(u_{n}\right), u_{n}\right\rangle \\
= & \left(\frac{1}{2}-\frac{1}{\theta}\right) a\left\|u_{n}\right\|^{2}+\left(\frac{1}{4-s}-\frac{1}{\theta}\right) b\left\|u_{n}\right\|^{4-s} \\
& +\left(\frac{1}{\theta}-\frac{1}{2^{*}(s)}\right) \int_{\Omega} \frac{|u|^{2 *(s)}}{|x|^{s}} \mathrm{~d} x+\int_{\Omega}\left(\frac{1}{\theta} f\left(x, u_{n}\right) u_{n}-F\left(x, u_{n}\right)\right) \mathrm{d} x \\
\geq & \left(\frac{1}{2}-\frac{1}{\theta}\right) a\left\|u_{n}\right\|^{2},
\end{aligned}
$$

where $\theta=\min \left\{\rho, 2^{*}(s)\right\}$. Thus we conclude that $\left\{u_{n}\right\}$ is a bounded sequence in $H_{0}^{1}(\Omega)$. By the continuity of embedding we have $\left|u_{n}\right|_{2^{*}(s)}^{2^{*}(s)} \leq C_{1}<\infty$ (denoting the usual $L^{p}(\Omega)$ norm by $\left.|\cdot|_{p}\right)$. Up to subsequences if necessary, there exists $u \in H_{0}^{1}(\Omega)$ such that

$$
\begin{array}{ll}
u_{n} \rightarrow u & \text { weakly in } H_{0}^{1}(\Omega), \\
u_{n} \rightarrow u & \text { in } L^{q}(\Omega) \text { for } q \in\left[1,2^{*}(s)\right], \\
u_{n} \rightarrow u & \text { a.e. in } \Omega .
\end{array}
$$

Then we prove that $u_{n} \rightarrow u$ in $H_{0}^{1}(\Omega)$.

By $\left(f_{1}\right)$, for any $\epsilon>0$, there exists $a(\epsilon)$ such that

$$
f(x, t) \leq \frac{1}{2 C_{1}} \epsilon t^{5-2 s}+a(\epsilon) .
$$

Let $\delta_{1}=\frac{\epsilon}{2 a(\epsilon)}$. When $E \subset \Omega$ and mes $E<\delta_{1}$, we have

$$
\begin{aligned}
\left|\int_{E} f\left(x, u_{n}\right) u_{n} \mathrm{~d} x\right| & \leq \int_{E}\left|f\left(x, u_{n}\right) u_{n}\right| \mathrm{d} x \\
& \leq \int_{E} a(\epsilon) \mathrm{d} x+\frac{1}{2 C_{1}} \epsilon \int_{E}\left|u_{n}\right|^{2^{*}(s)} \mathrm{d} x \\
& \leq a(\epsilon) \operatorname{mes} E+\frac{1}{2 C_{1}} \epsilon C_{1} \\
& \leq \epsilon .
\end{aligned}
$$


Hence $\left\{\int_{\Omega} f\left(x, u_{n}\right) u_{n} \mathrm{~d} x, n \in N\right\}$ is equiabsolutely continuous. It is easy to get the following from the Vitali convergence theorem:

$$
\int_{\Omega} f\left(x, u_{n}\right) u_{n} \mathrm{~d} x \rightarrow \int_{\Omega} f(x, u) u \mathrm{~d} x \quad \text { as } n \rightarrow \infty .
$$

Similarly, we can prove that

$$
\int_{\Omega} F\left(x, u_{n}\right) \mathrm{d} x \rightarrow \int_{\Omega} F(x, u) \mathrm{d} x \quad \text { as } n \rightarrow \infty .
$$

Further, by the concentration compactness principle [15] there exist a countable set $\Gamma$, a set of different points $\left\{x_{j}\right\} \subset \Omega \backslash\{0\}$, nonnegative real numbers $\mu_{x_{j}}, v_{x_{j}}$ for $j \in \Gamma$, and nonnegative real numbers $\mu_{0}, \gamma_{0}, v_{0}$ such that

$$
\begin{aligned}
& \left|\nabla u_{n}\right|^{2} \rightarrow \mathrm{d} \tilde{\mu} \geq|\nabla u|^{2}+\sum_{j \in \Gamma} \mu_{x_{j}} \delta_{x_{j}}+\mu_{0} \delta_{0}, \\
& u_{n}^{2}|x|^{-2} \rightarrow \mathrm{d} \gamma=u^{2}|x|^{-2}+\gamma_{0} \delta_{0}, \\
& \left|u_{n}\right|^{2^{*}(s)}|x|^{-s} \rightarrow \mathrm{d} v=|u|^{2^{*}(s)}|x|^{-s}+\sum_{j \in \Gamma} v_{x_{j}} \delta_{x_{j}}+v_{0} \delta_{0},
\end{aligned}
$$

where $\delta_{x}$ is the Dirac mass at $x \in \Omega$. For any $\epsilon>0$, we let $x_{j} \notin B_{\epsilon}(0)$ for all $j \in \Gamma$ and choose $\phi$ to be s smooth cut-off function such that $0 \leq \phi \leq 1, \phi \equiv 0$ for $x \in B_{\epsilon}^{c}(0), \phi \equiv 1$ for $x \in B_{\epsilon / 2}(0)$, and $|\nabla \phi| \leq 4 / \epsilon$. Then

$$
\begin{aligned}
& \lim _{\epsilon \rightarrow 0} \lim _{n \rightarrow \infty} \int_{\Omega}\left|\nabla u_{n}\right|^{2} \phi \mathrm{d} x=\lim _{\epsilon \rightarrow 0} \int_{\Omega} \phi \mathrm{d} \tilde{\mu} \geq \mu_{0}, \\
& \lim _{\epsilon \rightarrow 0} \lim _{n \rightarrow \infty} \int_{\Omega} u_{n}^{2}|x|^{-2} \phi \mathrm{d} x=\lim _{\epsilon \rightarrow 0} \int_{\Omega} \phi \mathrm{d} \gamma=\gamma_{0}, \\
& \lim _{\epsilon \rightarrow 0} \lim _{n \rightarrow \infty} \int_{\Omega}\left|u_{n}\right|^{2^{*}(s)}|x|^{-s} \phi \mathrm{d} x=\lim _{\epsilon \rightarrow 0} \int_{\Omega} \phi \mathrm{d} \nu=v_{0}, \\
& \lim _{\epsilon \rightarrow 0} \lim _{n \rightarrow \infty} \int_{\Omega}\left(\nabla u_{n} \nabla \phi\right) u_{n} \mathrm{~d} x=0, \\
& \lim _{\epsilon \rightarrow 0} \lim _{n \rightarrow \infty} \int_{\Omega} f\left(x, u_{n}\right) u_{n} \phi \mathrm{d} x=0 .
\end{aligned}
$$

The proofs of (3.4) and (3.5) are similar to that of Theorem 2.3 in [8] and are omitted here. Since $\left\{u_{n}\right\}$ is bounded, by (3.1) we have

$$
\begin{aligned}
0= & \lim _{\epsilon \rightarrow 0} \lim _{n \rightarrow \infty}\left\langle I^{\prime}\left(u_{n}\right), u_{n} \phi\right\rangle \\
= & \lim _{\epsilon \rightarrow 0} \lim _{n \rightarrow \infty}\left\{\left(a+b\left\|u_{n}\right\|^{2-s}\right)\left[\int_{\Omega}\left(\left|\nabla u_{n}\right|^{2} \phi+\left(\nabla u_{n} \nabla \phi\right) u_{n}-\mu \frac{u_{n}^{2}}{|x|^{2}} \phi\right) \mathrm{d} x\right]\right. \\
& \left.-\int_{\Omega} \frac{\left|u_{n}\right|^{2^{*}(s)}}{|x|^{s}} \phi \mathrm{d} x-\int_{\Omega} f\left(x, u_{n}\right) u_{n} \phi \mathrm{d} x\right\} \\
\geq & a\left(\mu_{0}-\mu \gamma_{0}\right)+b\left(\mu_{0}-\mu \gamma_{0}\right)^{(4-s) / 2}-v_{0} .
\end{aligned}
$$


Combining this with (2.1), we have that $S^{2^{*}(s) / 2} \nu_{0} \leq\left(\mu_{0}-\mu \gamma_{0}\right)^{2^{*}(s) / 2}$, and we deduce that

$$
S^{-3+s}\left(\mu_{0}-\mu \gamma_{0}\right)^{2-s}-b\left(\mu_{0}-\mu \gamma_{0}\right)^{(2-s) / 2}-a \geq 0
$$

which implies

$$
\left(\mu_{0}-\mu \gamma_{0}\right) \geq S\left[\frac{b S^{\frac{4-s}{2}}+\sqrt{b^{2} S^{4-s}+4 a S}}{2}\right]^{\frac{2}{2-s}} .
$$

Therefore we get

$$
\begin{aligned}
c+o(1)= & I\left(u_{n}\right)-\frac{1}{4-s}\left\langle I^{\prime}\left(u_{n}\right), u_{n}\right\rangle \\
= & \frac{a(2-s)}{2(4-s)}\left\|u_{n}\right\|^{2}+\frac{2-s}{2(3-s)(4-s)} \int_{\Omega} \frac{\left|u_{n}\right|^{2^{*}(s)}}{|x|^{s}} \mathrm{~d} x \\
& +\frac{1}{4-s} \int_{\Omega} f\left(x, u_{n}\right) u_{n} \mathrm{~d} x-\int_{\Omega} F\left(x, u_{n}\right) \mathrm{d} x \\
\geq & \frac{a(2-s)}{2(4-s)}\left(\mu_{0}-\mu \gamma_{0}\right)+\frac{2-s}{2(3-s)(4-s)} v_{0} \\
\geq & \frac{a(2-s)}{2(4-s)}\left(\mu_{0}-\mu \gamma_{0}\right)+\frac{2-s}{2(3-s)(4-s)}\left[a\left(\mu_{0}-\mu \gamma_{0}\right)+b\left(\mu_{0}-\mu \gamma_{0}\right)^{(4-s) / 2}\right] \\
\geq & \Lambda
\end{aligned}
$$

a contradiction. Thus we obtain

$$
\int_{\Omega} \frac{\left|u_{n}\right|^{2^{*}(s)}}{|x|^{s}} \mathrm{~d} x \rightarrow \int_{\Omega} \frac{|u|^{2^{*}(s)}}{|x|^{s}} \mathrm{~d} x .
$$

Combining this with (3.2), we find

$$
\begin{aligned}
o(1) & =\left\langle I^{\prime}\left(u_{n}\right)-I^{\prime}(u), u_{n}-u\right\rangle \\
& =\left(a+b\left\|u_{n}\right\|^{2-s}\right)\left(u_{n}, u_{n}-u\right)-\left(a+b\|u\|^{2-s}\right)\left(u, u_{n}-u\right)+o(1) \\
& =\left(a+b\left\|u_{n}\right\|^{2-s}\right)\left(u_{n}-u, u_{n}-u\right)+b\left(\left\|u_{n}\right\|^{2-s}-\|u\|^{2-s}\right)\left(u, u_{n}-u\right)+o(1) \\
& \geq a\left\|u_{n}-u\right\|^{2}+o(1),
\end{aligned}
$$

which shows that $u_{n} \rightarrow u$ in $H_{0}^{1}(\Omega)$. The proof is completed.

Lemma 3.2 If $f(x, t)$ satisfies $\left(f_{1}\right)$ and $\left(f_{2}\right)$, then there exists $u_{0} \in H_{0}^{1}(\Omega)$ such that

$$
\sup _{t \geq 0} I\left(t u_{0}\right)<\Lambda
$$

Proof We consider the functions

$$
\begin{aligned}
& g(t)=\frac{a t^{2}}{2}\left\|v_{\epsilon}\right\|^{2}+\frac{b t^{4-s}}{4-s}\left\|v_{\epsilon}\right\|^{4-s}-\frac{t^{2^{*}(s)}}{2^{*}(s)}-\int_{\Omega} F\left(x, t v_{\epsilon}\right) \mathrm{d} x, \\
& g_{0}(t)=\frac{a t^{2}}{2}\left\|v_{\epsilon}\right\|^{2}+\frac{b t^{4-s}}{4-s}\left\|v_{\epsilon}\right\|^{4-s}-\frac{t^{2^{*}(s)}}{2^{*}(s)} .
\end{aligned}
$$


From

$$
0=g^{\prime}\left(t_{\epsilon}\right)=a t_{\epsilon}\left\|v_{\epsilon}\right\|^{2}+b t_{\epsilon}^{3-s}\left\|v_{\epsilon}\right\|^{4-s}-t_{\epsilon}^{2^{*}(s)-1}-\int_{\Omega} f\left(x, t_{\epsilon} v_{\epsilon}\right) v_{\epsilon} \mathrm{d} x
$$

we derive

$$
a\left\|v_{\epsilon}\right\|^{2}+b t_{\epsilon}^{2-s}\left\|v_{\epsilon}\right\|^{4-s}=t_{\epsilon}^{4-2 s}+\frac{1}{t_{\epsilon}} \int_{\Omega} f\left(x, t_{\epsilon} v_{\epsilon}\right) v_{\epsilon} \mathrm{d} x \geq t_{\epsilon}^{4-2 s} .
$$

Since $4-2 s=2(2-s)$, we have

$$
t_{\epsilon} \leq\left[\frac{b\left\|v_{\epsilon}\right\|^{4-s}+\sqrt{b^{2}\left\|v_{\epsilon}\right\|^{2(4-s)}+4 a\left\|v_{\epsilon}\right\|^{2}}}{2}\right]^{\frac{1}{2-s}} \triangleq t_{0}
$$

By $\left(f_{1}\right)$, obviously,

$$
f(x, t) \leq \epsilon t^{5-2 s}+d(\epsilon) t, \quad d(\epsilon)>0
$$

Therefore we obtain

$$
a\left\|v_{\epsilon}\right\|^{2}+b t_{\epsilon}^{2-s}\left\|v_{\epsilon}\right\|^{4-s} \leq t_{\epsilon}^{4-2 s}+\epsilon \int_{\Omega}\left|t_{\epsilon}\right|^{4-2 s}\left|v_{\epsilon}\right|^{2^{*}(s)} \mathrm{d} x+d(\epsilon) \int_{\Omega}\left|v_{\epsilon}\right|^{2} \mathrm{~d} x
$$

and

$$
t_{\epsilon}^{4-2 s}+\epsilon \int_{\Omega}\left|t_{\epsilon}\right|^{4-2 s}\left|v_{\epsilon}\right|^{2^{*}(s)} \mathrm{d} x=t_{\epsilon}^{4-2 s}\left(1+\epsilon \int_{\Omega}\left|v_{\epsilon}\right|^{2^{*}(s)} \mathrm{d} x\right) \leq \frac{3}{2} t_{\epsilon}^{4-2 s} .
$$

Thanks to (2.3), when $\epsilon$ is small enough, we conclude from $d(\epsilon) \int_{\Omega}\left|v_{\epsilon}\right|^{2} \mathrm{~d} x \rightarrow 0$ as $\epsilon \rightarrow 0$ that

$$
d(\epsilon) \int_{\Omega}\left|v_{\epsilon}\right|^{2} \mathrm{~d} x \leq a\left\|v_{\epsilon}\right\|^{2} .
$$

From (3.7)-(3.9) we get

$$
a\left\|v_{\epsilon}\right\|^{2}+b t_{\epsilon}^{2-s}\left\|v_{\epsilon}\right\|^{4-s} \leq \frac{3}{2} t_{\epsilon}^{4-2 s}+a\left\|v_{\epsilon}\right\|^{2} .
$$

Combining this with (2.2), we obtain

$$
b S^{\frac{4-s}{2}} \leq b\left\|v_{\epsilon}\right\|^{4-s} \leq \frac{3}{2} t_{\epsilon}^{2-s}
$$

which implies

$$
t_{\epsilon} \geq\left(\frac{2 b S^{\frac{4-s}{2}}}{3}\right)^{\frac{1}{2-s}}
$$

Consequently, the function $g_{0}(t)$ attains its maximum at $t_{0}$ and continuously increases in the interval $\left[0, t_{0}\right]$. From this, together with (2.2) and the inequality $F(x, t) \geq C_{2}|t|^{\rho}$, which 
is directly obtained from $\left(f_{2}\right)$, we derive that

$$
\begin{aligned}
g(t) \leq & g_{0}\left(t_{0}\right)-\int_{\Omega} F\left(x, t_{\epsilon} v_{\epsilon}\right) \mathrm{d} x \\
= & \frac{a(2-s)}{2(3-s)} t_{0}^{2}\left\|v_{\epsilon}\right\|^{2}+\frac{b(2-s)}{2(3-s)(4-s)^{4-s}} t^{4-s} v_{\epsilon} \|^{4-s}-\int_{\Omega} F\left(x, t_{0} v_{\epsilon}\right) \mathrm{d} x \\
\leq & \frac{a(2-s)}{2(3-s)}\left\|v_{\epsilon}\right\|^{2}\left[\frac{b\left\|v_{\epsilon}\right\|^{4-s}+\sqrt{b^{2}\left\|v_{\epsilon}\right\|^{2(4-s)}+4 a\left\|v_{\epsilon}\right\|^{2}}}{2}\right]^{\frac{2}{2-s}} \\
& +\frac{b(2-s)}{2(3-s)(4-s)}\left\|v_{\epsilon}\right\|^{4-s}\left[\frac{b\left\|v_{\epsilon}\right\|^{4-s}+\sqrt{b^{2}\left\|v_{\epsilon}\right\|^{2(4-s)}+4 a\left\|v_{\epsilon}\right\|^{2}}}{2}\right]^{\frac{4-s}{2-s}} \\
& -C_{2} \int_{\Omega} t_{\epsilon}^{\rho}\left|v_{\epsilon}\right|^{\rho} \mathrm{d} x \\
\leq & \Lambda+O\left(\frac{1}{2(2-s)}\right)-C_{2}\left(\frac{2 b S^{\frac{4-s}{2}}}{3}\right)^{\frac{\rho}{2-s}} \int_{\Omega}\left|v_{\epsilon}\right|^{\rho} \mathrm{d} x .
\end{aligned}
$$

In addition, from (2.3) it follows that

$$
\int_{\Omega}\left|v_{\epsilon}\right|^{\rho} \mathrm{d} x=O\left(\epsilon^{\frac{\sqrt{\mu}(3-\rho \sqrt{\mu})}{(2-s) \sqrt{\mu}-\mu}}\right) .
$$

Thanks to $\left(f_{2}\right)$, we have

$$
\frac{1}{2(2-s)}>\frac{\sqrt{\bar{\mu}}(3-\rho \sqrt{\bar{\mu}})}{(2-s) \sqrt{\bar{\mu}-\mu}} .
$$

Choosing $\epsilon$ small enough, we conclude

$$
\sup _{t \geq 0} I\left(t v_{\epsilon}\right)=g\left(t_{\epsilon}\right)<\Lambda .
$$

This completes the proof of Lemma 3.2.

Next, we prove that the functional $I(u)$ satisfies the mountain pass geometry.

Lemma 3.3 Suppose that $\left(f_{1}\right)$ and $\left(f_{2}\right)$ hold. Then we have:

(i) there exist $r, \beta>0$ such that $\inf _{\|u\|=r} I(u) \geq \beta>0$,

(ii) there exists a nonnegative function $e \in H_{0}^{1}(\Omega)$ such that $\|e\|>r$ and $I(e)<0$.

Proof (i) By $\left(f_{1}\right)$, for any $\epsilon>0$, there exists $C_{3}$ such that

$$
|f(x, t)| \leq \epsilon t+C_{3} t^{5-2 s}
$$

for all $t \in \mathbb{R}^{+}$and $x \in \bar{\Omega}$. By the definition of $F(x, u)$ we get

$$
|F(x, t)| \leq \frac{1}{2} \epsilon t^{2}+C_{4} t^{2^{*}(s)}
$$


for all $t \in \mathbb{R}^{+}$and $x \in \bar{\Omega}$, where $C_{5}=\frac{1}{2^{*}(s)} C_{3}$. Then by (2.1),we get

$$
\begin{aligned}
I(u) & =\frac{a}{2}\|u\|^{2}+\frac{b}{4-s}\|u\|^{4-s}-\frac{1}{2^{*}(s)} \int_{\Omega} \frac{|u|^{2^{*}(s)}}{|x|^{s}} \mathrm{~d} x-\int_{\Omega} F(x, u) \mathrm{d} x \\
& \geq \frac{a}{2}\|u\|^{2}-\frac{1}{2^{*}(s)} S^{-\frac{2^{*}(s)}{2}}\|u\|^{2^{*}(s)}-\frac{1}{2} \epsilon|u|_{2}^{2}-C_{5}|u|_{2^{*}(s)}^{2^{*}(s)} \\
& \geq \frac{a}{2}\|u\|^{2}-\frac{1}{2^{*}(s)} S^{-\frac{2^{*}(s)}{2}}\|u\|^{2^{*}(s)}-\frac{C}{2} \epsilon\|u\|_{2}^{2}-C C_{5}\|u\|_{2^{*}(s)}^{2^{*}(s)}
\end{aligned}
$$

for $\epsilon$ small enough. Hence there exists $\beta>0$ such that $I(u) \geq \beta$ for all $\|u\|=r$, where $r>0$ is small enough.

By Lemma 3.2, there exists $u_{0} \in H_{0}^{1}(\Omega), u_{0} \not \equiv 0$ such that

$$
\sup _{t \geq 0} I\left(t u_{0}\right)<\Lambda
$$

It follows from the nonnegativity of $F(x, t)$ that

$$
\begin{aligned}
I\left(t u_{0}\right) & =\frac{a t^{2}}{2}\left\|u_{0}\right\|^{2}+\frac{b t^{4-s}}{4-s}\left\|u_{0}\right\|^{4-s}-\frac{t^{2^{*}(s)}}{2^{*}(s)} \int_{\Omega} \frac{\left|u_{0}\right|^{2^{*}(s)}}{|x|^{s}} \mathrm{~d} x-\int_{\Omega} F\left(x, t u_{0}\right) \mathrm{d} x \\
& \leq \frac{a t^{2}}{2}\left\|u_{0}\right\|^{2}+\frac{b t^{4-s}}{4-s}\left\|u_{0}\right\|^{4-s}-\frac{t^{2^{*}(s)}}{2^{*}(s)} \int_{\Omega} \frac{\left|u_{0}\right|^{2^{*}(s)}}{|x|^{s}} \mathrm{~d} x,
\end{aligned}
$$

which shows that $\lim _{t \rightarrow+\infty} I\left(t u_{0}\right) \rightarrow-\infty$. Therefore we can choose $t_{0}$ such that $\left\|t_{0} u_{0}\right\|>r$ and $I\left(t_{0} u_{0}\right) \leq 0$. The proof of Lemma 3.3 is completed.

Proof of Theorem 1.1 By the mountain pass theorem in [16] there is a sequence $\left\{u_{n}\right\} \subset$ $H_{0}^{1}(\Omega)$ satisfying

$$
I\left(u_{n}\right) \rightarrow c \geq \beta \quad \text { and } \quad I^{\prime}\left(u_{n}\right) \rightarrow 0,
$$

where

$$
\begin{aligned}
& c=\inf _{\gamma \in \tau} \max _{t \in[0,1]} I(\gamma(t)), \\
& \tau=\left\{\gamma \in C\left([0,1], H_{0}^{1}(\Omega)\right) \mid \gamma(0)=0, \gamma(1)=t_{0} u_{0}\right\} .
\end{aligned}
$$

Therefore

$$
0<\beta \leq c=\inf _{\gamma \in \tau} \max _{t \in[0,1]} I(\gamma(t)) \leq \max _{t \in[0,1]} I\left(t t_{0} u_{0}\right) \leq \sup _{t \geq 0} I\left(t u_{0}\right)<\Lambda .
$$

Applying this inequality and Lemma 3.1, we can obtain a critical point $u_{1}$ of the functional $I$. From the continuity of $I^{\prime}$ we deduce that $u_{1}$ is a weak solution of problem (1.1). Then $\left\langle I^{\prime}\left(u_{1}\right), u_{1}^{-}\right\rangle=0$, where $u_{1}^{-}=\min \{u, 0\}$. Thus $u_{1} \geq 0$ and $u_{1} \not \equiv 0$. By the strong maximum principle there is $u_{1}>0$ that is a positive solution of problem (1.1). Thus Theorem 1.1 holds.

Proof of Theorem 1.2 Theorem 1.2 can be proved similarly. 


\section{Acknowledgements}

The authors thank the anonymous referee for his/her positive and useful comments, which helped him improve further the exposition of the paper.

\section{Funding}

This paper is supported by the National Natural Science Foundation of China (grant No. 11971339), the Chongqing research and innovation project of graduate students (grant No. CYS20271), and Science and Technology Research Project of Chongqing Education Commission (No. KJQN201800636).

\section{Availability of data and materials}

Not applicable.

\section{Competing interests}

The authors declare that they have no competing interests.

\section{Authors' contributions}

The main idea of this paper was proposed by HF and ZD. HF prepared the manuscript initially and performed all the steps of the proofs in this research. All authors read and approved the final manuscript.

\section{Publisher's Note}

Springer Nature remains neutral with regard to jurisdictional claims in published maps and institutional affiliations.

\section{Received: 27 July 2020 Accepted: 15 April 2021 Published online: 04 May 2021}

\section{References}

1. Kirchhoff, G.: Mechanik. Leipzig, Teuhner (1883)

2. Lions, J.L.: On some questions in boundary value problems of mathematical physics. North-Holl. Math. Stud. 30 284-346 (1978)

3. Jin, J.H., Wu, X.: Infinitely many radial solutions for Kirchhoff type problem in $\mathbb{R}^{N}$. J. Math. Anal. Appl. 369(2), 564-574 (2010)

4. Shao, L.Y., Chen, H.B.: Existence of solutions for the Schrödinger-Kirchhoff-Poisson systems with a critical nonlinearity. Bound. Value Probl. 2016, 210 (2016)

5. Chen, C.Y., Kuo, Y.C., Wu, T.F.: The Nehari manifold for a Kirchhoff type problem involving sign-changing weight functions. Int. J. Differ. Equ. 250, 1876-1908 (2011)

6. Xie, Q.L., Wu, X.P., Tang, C.L.: Existence and multiplicity of solutions for Kirchhoff type problem with critical exponent Commun. Pure Appl. Anal. 12(6), 2773-2786 (2013)

7. Lei, J.S.: Multiplicity and asymptotic behavior of solutions for Kirchhoff type equations involving the Hardy-Sobolev exponent and singular nonlinearity. J. Inequal. Appl. 2018, 213 (2018)

8. Naimen, D.: Positive solutions of Kirchhoff type elliptic equations involving a critical Sobolev exponent. Nonlinear Differ. Equ. Appl. 21(6), 885-914 (2014)

9. Ding, L., Tang, C.L.: Existence and multiplicity of solutions for semilinear elliptic equations with Hardy terms and Hardy-Sobolev critical exponents. Appl. Math. Lett. 20, 1175-1183 (2007)

10. Xu, L.P., Chen, H.B.: Ground state solutions for Kirchhoff-type equations with a general nonlinearity in the critical growth. Adv. Nonlinear Anal. 7(4), 535-546 (2018)

11. Fiscella, A., Pucci, P., Zhang, B.L.: p-fractional Hardy-Schrödinger-Kirchhoff systems with critical nonlinearities. Adv. Nonlinear Anal. 8, 1111-1131 (2019)

12. Qi, X.M., Radulescu, V.D., Zhang, B.L.: Combined effects for fractional Schrödinger Kirchhoff systems with critical nonlinearities. ESAIM Control Optim. Calc. Var. 24, 1249-1273 (2018)

13. Kufner, A., Persson, L.: Weighted Inequalities of Hardy Type. World Scientific, Singapore (2003)

14. Kang, D.S., Peng, S.: Positive solutions for singular critical elliptic problems. Appl. Math. Lett. 17, 411-416 (2004)

15. Lions, P.L.: The concentration-compactness principle in the calculus of variation. The locally compact case, Part I. Ann. Inst. Henri Poincaré, Anal. Non Linéaire 1(2), 109-145 (1984)

16. Ambrosetti, A., Rabinowitz, P.: Dual variational methods in critical point theory and applications. J. Appl. Funct. Anal. 14(4), 349-381 (1973) 